\title{
Closing the GAP Between Organizational Requirements and Object Oriented Modeling
}

\author{
Jaelson Castro \\ Universidade Federal de \\ Pernambuco, Centro de \\ Informática, Recife, Brazil \\ jbc@cin.ufpe.br
}

\author{
Fernanda Alencar \\ Universidade Federal de \\ Pernambuco, Dep. Eletrônica e \\ Sistemas, Recife, Brazil \\ fmra@npd.ufpe.br
}

\author{
Gilberto Cysneiros \\ Universidade Federal de \\ Pernambuco, Centro de \\ Informática, Recife, Brazil \\ gaacf@cin.ufpe.br
}

\begin{abstract}
Requirements Engineering has been considered a key activity in any Software Engineering process. It is well known that a requirements specification should include not only software specifications but also business models and other kinds of information describing the context in which the intended system will function. In recent years we have observed a growing influence of the object-orientation paradigm. Unfortunately, the current dominant object oriented modeling technique, i.e. Unified Modeling Technique, is ill equipped for modeling early requirements which are typically informal and often focus on Organizational objectives. UML is more suitable for later phases of requirements capture, which usually focus on completeness, consistency, and automated verification of functional requirements for the new system. In this paper, we present some guidelines for the integration of early and late requirements specifications. For the organizational modeling we use the $i^{*}$ framework, which focuses on the description of organizational relationships among various organizational actors, as well as an understanding of the rationale for the alternatives chosen. For the functional requirements specification, we rely on a precise subset of the Unified Modeling Language annotated with constraints described in the Object Constraint Language. A small example is used to illustrate how the requirements process iterates between the early and late requirements specification.
\end{abstract}

Keywords: Requirements Engineering, Object Oriented Development, UML

\section{Introduction}

It is now a widely shared tenet of Software Engineering that a good Requirements Engineering effort is paramount to the success of any system. Often, software systems fail to properly support the organizations of which they are an integral part. Primary reasons for such failures are the lack of proper understanding of the organization by the software developers of the system, also the frequency of organizational changes which cannot be accommodated by existing software systems (or their maintainers). Hence, requirements capture has been acknowledged as a critical phase of software development, precisely because it is the phase which deals not only with technical knowledge, but also with Organizational, managerial, economic and social issues.
The emerging consensus is that a requirement specification should include not only software specifications but also business models and other kinds of information describing the context in which the intended system will function [1]. During requirements analysis, analysts need to help to identify different ways in which software systems ca be used to achieve Organizational objectives. Consequently, there is a need for modeling and analysis of stakeholder interests and how they might be addressed, or compromised, by various system-andenvironment alternative structures. Indeed, the Unified Method has suggested the following archetypal workflow for requirements capture [2]:

- List candidate requirements,

- Understand system context, 
- Capture functional requirements,

- Capture non-functional requirements.

However, the production of high quality specifications is quite difficult. Usually the customers do not exactly know what they want and sometimes the requirements may not reflect the real needs of the customers. It is common for requirements to be incomplete and/or inconsistent.

A recent work on requirements engineering has drawn an important distinction between early phase requirements capture and late phase requirements capture [3]. Early phase requirements activities are typically informal and address Organizational or non-functional requirements. The emphasis is on understanding the motivation and rationale that underlie system requirements. Late phase requirements activities usually focus on completeness, consistency, and automated verification of requirements.

We claim that the dominant object oriented modeling technique, Unified Modeling Language [4], is well suited only for late-phase requirements capture. We argue that $\mathrm{UML}$ is ill equipped for early requirements capture because it can not represent how the intended system meets organizational goals, why the system is needed, what alternatives were considered, what the implication of the alternatives are for the various stakeholders, and how the stakeholders' interests and concerns might be addressed. What is required to capture such concerns is a framework that focuses on the description and evaluation of alternatives and their relationship to the organizational objectives behind the software development project [5]. We argue that the $i$ * framework [6], is well suited for early-phase requirements capture, since it provides for the representation of alternatives, and offers primitive modeling concepts such as those of softgoal and goal.

We advocate that UML alone is not adequate to deal with all different types of analysis and reasoning that are required during the requirements capture phases. Instead, we advocate the use of two complementary modeling techniques, $i^{*}$ and $U M L$. To model and understand issues of the application and business domain (the enterprise) a developer can use the $i^{*}$ framework which allows a better description of the organizational relationships among the various agents of a system as well as an understanding of the rationale of the decisions taken. For the functional requirements specification, the developer can rely on UML, or if formality is required, the precise Unified Modeling Language (pUML) [7], annotated with constraints described in OCL [8].

In this work we present the transition from early (informal) descriptions in $i^{*}$ to late (precise) requirements in $\mathrm{p} \boldsymbol{U} \boldsymbol{M L}$. This constitutes a conceptualization activity within which a developer might make use of domain knowledge partly expressed in descriptions of the organization, and partly in existing requirements specifications.

This paper is organized as follows. Section 2 introduces the language used for the early requirements description, namely the $i^{*}$ technique. In section 3 , we provide some means for transforming the $i *$ models into precise specifications in pUML/OCL. Late requirements specification is described in Section 4. Section 5 reviews some related work, while Section 6 concludes the paper with a summary of its contributions. Throughout the paper, a small CD store example is used to illustrate how the requirements process iterates between the early and late requirements specification. The example is pedagogical and is meant to be suggestive of the much more complex sets of issues typically found in actual situations

\section{The i* Modeling Framework for Early Requirements Capture}

When developing systems, we usually need to have a broad understanding of the organizational environment and goals. The $i^{*}$ technique [3] provides understanding of the "why" by modeling organizational relationships that underlie systems requirements. $i *$ offers a modeling framework that focuses on strategic actor relationships. Usually, when we try to understand an organization, the information captured by standard modeling techniques (DFD, ER, Statechart, etc.) focuses on entities, functions, data flows, states and the like. They are not capable of expressing the reasons (the "why's") of the process (motivations, intentions and rationales). The ontology of $i^{*}$ [9] caters to some of these more advanced concepts. It can be used for: (i) obtaining a better understanding of the organizational relationships among the various organizational agents; (ii) understanding the rationale of the decisions taken; and (iii) illustrating the various characteristics found in the early phases of requirements specification. The participants of the organizational setting are actors with intentional properties, such as, goals, beliefs, abilities and compromises. These actors depend upon each other in order to fulfil their objectives and have their tasks performed. The $i^{*}$ technique offers two models: The Strategic Dependency (SD) model, and the Strategic Rationale (SR) model.

\subsection{The Strategic Dependency Model}

This model focuses on the intentional relationships among organizational actors. It consists of a set of nodes and links connecting them, where nodes represent actors 
and each link indicates a dependency between two actors. The depending actor is called depender, and the actor who is depended upon is called the dependee. Hence, an SD model consists of a network of dependency relationships among various actors, capturing the motivation and the rationale of activities. $i^{*}$ distinguishes four types of dependencies. Three of these related to existing intentions - goal dependency, resource dependency and task dependency, while the fourth is associated with the notion of non-functional requirements, the so called softgoal dependency. In a goal dependency, an agent depends on another to fulfil a goal, without worrying how this goal will be achieved. In a resource dependency, an agent depends on another agent to provide a physical resource or information. In a task dependency, an agent depends on another to carry out a task. A softgoal dependency is similar to a goal dependency, except that a softgoal is not precisely defined. In $i^{*}$ we can also model different degrees of dependency commitment on the part of the relevant actors (open, committed, or critical). We can also classify actors into agents, roles and positions. An agent is an actor with concrete physical manifestations (a person or a system). A role is an abstract characterization of the behaviour of a social actor within some specialized context, domain or endeavor. A position is a set of roles typically played by one agent. Finally, $i^{*}$ supports the analysis of opportunities and vulnerabilities for different actors [6].

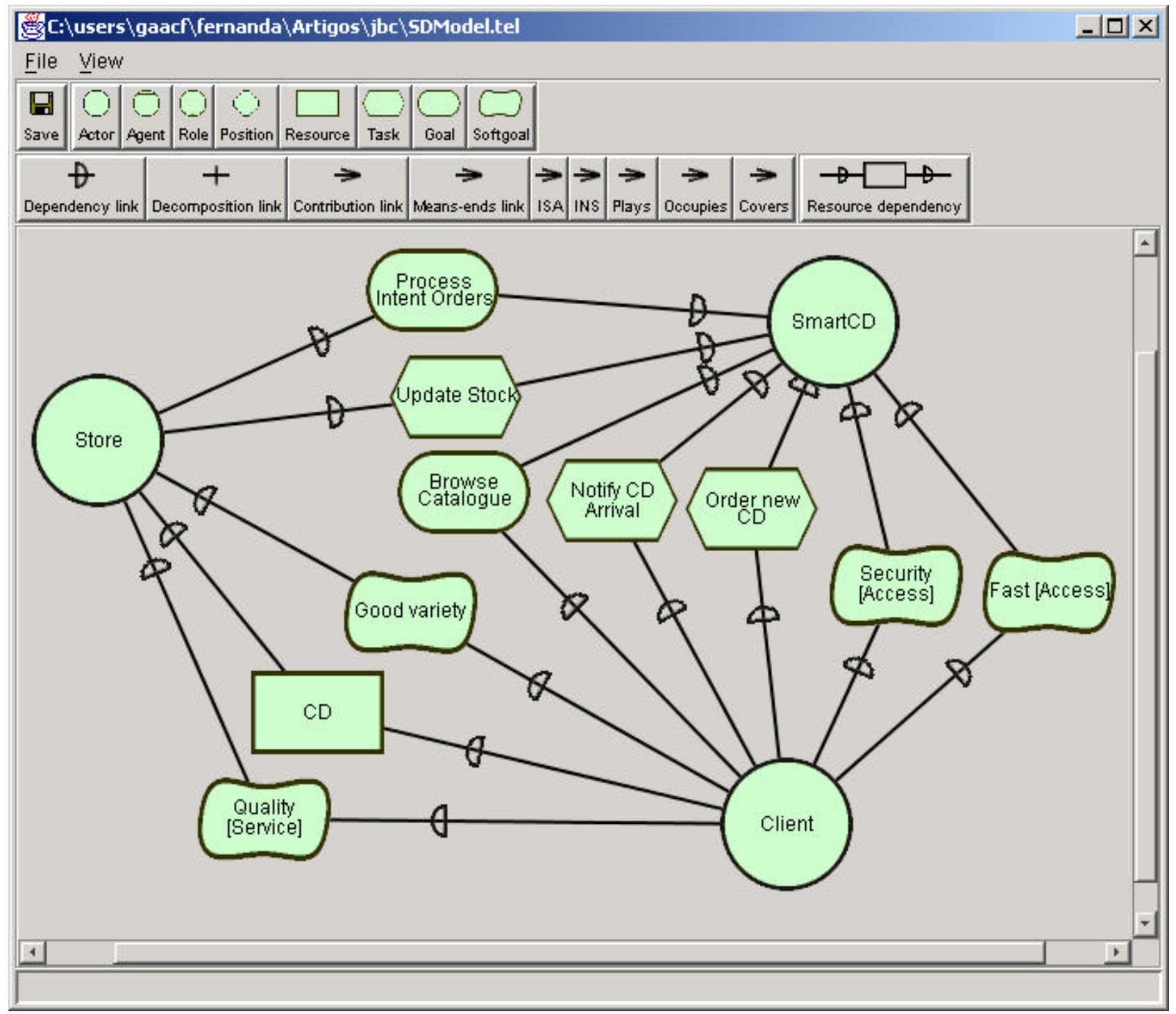

Figure 1: Strategic Dependency Model 
Suppose a situation in which a Client wishes to buy CDs and goes to a specialized store because its services are of good quality and it claims to have most (if not all) available titles on stock. If a client cannot find his/hers preferred title, the shop can happily place an order for it and notify the client upon its arrival. The shop has decided to improve its services by commissioning a new software system (SmartCD) to handle orders as well as providing an online catalogue (it would be so convenient!). In Figure 1, we have the Strategic Dependency (SD) model of the CD store case study.

At this early stage of requirements capture we have identified three actors: Client, Store and SmartCD. This last actor indeed corresponds to the system to be developed, handling orders, notifications of $\mathrm{CD}$ arrivals and providing the online catalogue. The dependencies between the Client and the Store actor can be find in Figure 1. The Client depends on the Store for getting the CD (resource dependency). However, he/she wishes the services to be of good quality (softgoal Quality[Service]) and the store to maintain a good stock of CDs (softgoal Good variety). Of course these goals are not yet precisely defined at this early stage, hence the use of softgoals. Turning to the relationship between actors Client and SmartCD, we notice that one of the goals for introducing the online system is to enable browsing facilities (goal dependency Browse Catalogue). In fact, the store may stock thousands of CDs, making it difficult (or even impossible) for a customer to manually search all of them. In the (unlikely) situation that a CD is not on stock, the SmartCD actor will be able to handle orders online (the system will inform what and how it should be done, hence task dependency Order new $C D$ ). This feature is much awaited, since filling orders manually (through a sales person) is time consuming. Of course, when the (ordered) CD arrives, the Client will be notified as soon as possible (actually there is a pre-defined procedure for dealing with it, hence the task dependency Notify $C D$ Arrival). The Client expects the access to software system to be fast (softgoal Fast[Access]) and to use it to keep the stock updated (task Update Stock). Last but not least, the Store actor also has some expectations on the commissioned SmartCD system. It relies on the software system to process internet orders (goal Process Internet Orders) and to controls its stock (task Update Stock).

\subsection{The Strategic Rational Model}

The Strategic Rationale Model (SR) provides a more detailed level of modeling by looking "inside" actors to model internal intentional relationships. It is used to: (i) describe the interests, concerns and motivations of participants process; (ii) enable the assessment of the possible alternatives in the definition of the process; and (iii) research in more detail the existing reasons behind the dependencies between the various actors. Nodes and links also compose this model. It includes the previous four types of nodes (present in the SD model): goal, task, resource and soft-goal. However, two new types of relationship are incorporated: means-end that suggests that there could be other means of achieving the objective (alternatives) and task-decomposition that describes what should be done in order to perform a certain task.

In Figure 2 we use the SR notation to detail the Store agent. Due to space limitation we now only comment some aspects. An interested reader can find a fuller description of the approach in [10]. The store is interested in attracting (new and old) clients (expressed by task node To Attract Customers). Several strategic decisions were taken in consideration and as a result the task was decomposed into five aspects (expressed by a taskdecomposition link):

- The need to offer reasonable prices (captured by software goal Good Prices). Two alternatives are considered for meeting this objective: to offer discount on selected items (softgoal Discount Price) or obtaining good deals by buying large quantities of popular CDs (softgoal Volume Purchase[Highs]),

- To need to establish a courteous relationship between the store staff and its clients (represented by softgoal Friendly Staff). This is considered to be a way of meeting the quality of service expected by the client.

- To define standard procedures for delivering CD (expressed by sub-task Delivery),

- To be able to maintain a good stock of CDs (captured by sub-task Maintain Stock). This will also require the updating of the online information system. Hence the task dependency Update Stock between the Store and SmartCD actors.

- To handle internet sales (captured by sub-task Sales by Web), which depends on the adequate software system, hence the goal dependency Process Internet Orders between the Store and SmartCD actors. 


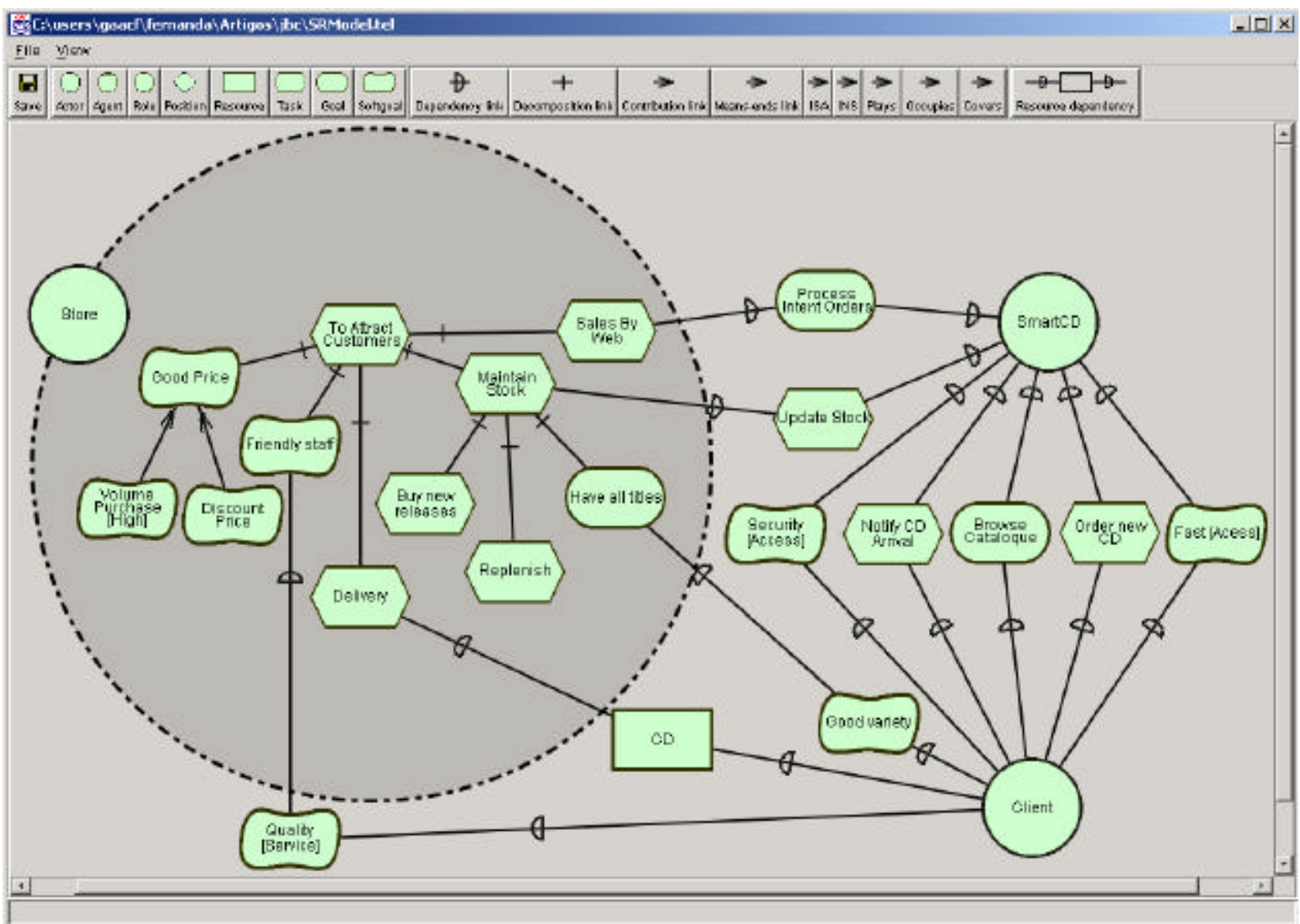

Figure 2: Strategic Rational Model

After some considerations, it has been agreed that the task of maintaining the stock needs to be further decomposed:

- The overall objective is to have all available titles on stock (captured by goal Have all titles). If this is met, certainly the client will be very pleased (see the softgoal Good Variety between Client and Store actors),

- Buying new titles (expressed by a sub-task Buy new releases),

- Making sure that popular CDs are re-stocked (expressed by a sub-task 'Replenish').

At this point, we may stop the process of modeling the strategic dependencies of the CD store. We are already capable of understanding some issues of the application domain (the enterprise). We can then move to provide a detailed system specification.

\section{From Organizational Requirements to Object Oriented Models}

We have shown that $i^{*}$ provides an early understanding of the organizational relationships in a business domain. As we progress in the development we need to focus on the functional and non-functional requirements of a system-to-be, which will support the chosen alternative among those considered during early requirements. To specify the late requirements, we adopt pUML (precise UML) [7] which provides a precise denotational semantics for core UML elements, such as: relationship, classifier, association, and generalization. The interested reader can visit the pUML site [11] for a complete description of the approach.

Note that pUML diagrams alone are not sufficient for late requirement capture because it does not provide for the specification of constraints, such as invariants, preconditions and the like. For this task, we have adopted the Object Constraint Language (OCL) [8]. OCL is a textual language, also part of the Object Man- 
agement standard, that can precisely describe constraints for object oriented models.

In the sequel we suggest six heuristics for transforming $i^{*}$ based early requirements models to pUML/OCL based late requirements:

- Guideline G1: Related to actors;

- Guideline G2: Related to tasks;

- Guideline G3: Related to resources;

- Guideline G4: Related to goals and soft-goals;

- Guideline G5: Related to tasks decomposition;

- Guideline G6: Related to means ends links.

\section{Guideline G1:}

Actors in the $i^{*}$ framework, can be mapped to classes in pUML. OCL constraints can be attached to the actorgenerated classes.

In our case study (see Figure 1) there were three actors: Store, Client and SmartCD. These actors can be mapped to the three classes shown in Figure 3.

\section{Guideline G1.1:}

Actor composition in $i^{*}$ corresponds to class aggregation in pUML.

In our CD Store case study (see Figure 1), the Strategic Dependency contained three actors: Store, Client and SmartCD. In pUML (see Figure 3), CD Store class is the aggregate of three composite classes.

\section{Guideline G2:}

Tasks in $i^{*}$, are mapped to class operations in pUML.

\section{Guideline G2.1:}

A task dependency, between a depender and a dependee actor in the SD model, corresponds to a public operation in the dependee pUML class.

In our case study (see Figure 1), the Store Actor depends on the SmartCD Actor for updating its stock (task Update Stock). Similarly, the Client actor depends on the SmartCD Actor for two tasks: ordering CDs (Order new $C D$ ) and receiving notification of goods arrival (task Notify CD arrivals). Hence, in Figure 3 you can observe that the SmartCD pUML class will be responsible for supporting the three (public) operations (Update Stock, Notify CD Arrival, Order New CD) .

\section{Guideline G2.2:}

A task in the SR model is mapped to a local operation in the corresponding pUML class.

In our case study, see Figure 2, a key task for the
Store actor was to be able to Attract Customers. This consists of three subtasks: to handle internet orders (Sales By Web), to Maintain Stock and to deliver the CDs (Delivery). Maintaining stock included obtaining the new releases (Buy new releases) and renewing items (Replenish). Therefore the corresponding Store class has five (local) corresponding operations (see Figure 3 ).

\section{Guideline G3:}

Resources in $i^{*}$ are mapped as classes in pUML. A public attribute of the type Boolean, indicates the availability of resource.

In our example, see Figure 1, Client actor depends on the Store Actor, to obtain a CD resource. In Figure 3, we can observe that the $C D$ class has been introduced to represent the resource. A boolean attribute (availability) indicates if is the resource is at hand.

\section{Guideline G4:}

Strategic goals and soft goals will be mapped to attributes of the type boolean and enumerated type, respectively, in pUML classes.

Goals are well defined, hence it is always possible to establish if one has been fulfilled or not. On the other hand, softgoals are not well defined. They can only be "satisfied" to some degree [16]. Hence, an enumerated type is better suited for their representation in pUML/OCL, whose values represent different degrees of softgoal fulfillment.

\section{Guideline G4.1:}

Goals and soft goals dependencies in Strategic Dependency Models (SD) models are mapped to public boolean and enumerated attributes, respectively, of the dependee pUML class.

In our case study (see Figure 1), the Client Actor expects that the Store Actor could have a good stock (Good variety softgoal) and provide a good service (Quality[Service] softgoal). Therefore, in the corresponding Store class, two enumerated public attributes are added (see Figure 3).

\section{Guideline G4.2:}

Goals and soft goals dependencies in Strategic Rationale Models (SR), are mapped to local boolean and enumerated pUML class attributes, respectively.

For example, in Figure 2, we have that the Store actor has a well defined goal (to Have all titles), and four ill defined objectives or softgoals: to offer Good Price, to have Volume Purchase [High], to give Discount Price, and to have a Friendly staff. In Figure 3 we observe that these extra attributes have been included to 


\section{Store Class.}

The next guideline deals with task. Operations in pUML can be used to describe tasks performed by an actor. If we need to provide a more precise account of the operation, we can rely on OCL to specify its pre and post conditions. However, observe that in the $i^{*}$ framework tasks can also be decomposed into sub-task, subgoal, sub-softgoal and sub-resource.

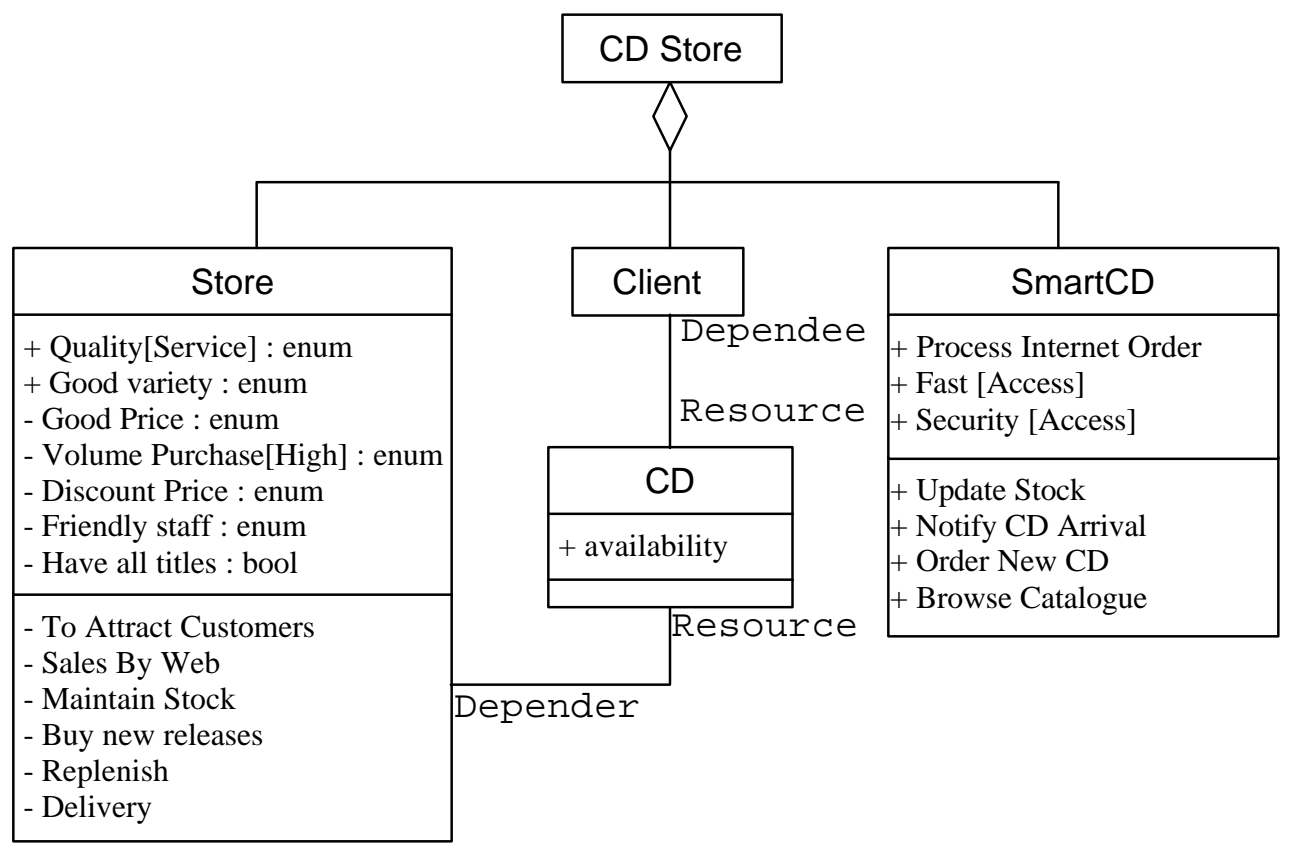

Figure 3: Context Class Diagram of the CD Store

\section{Guideline G5:}

Task decomposition is represented by pre and posconditions (expressed in OCL) of the corresponding pUML operation.

The pre-condition is the conjunction (AND OCL connector) of sub-tasks pre-conditions.

The post-condition is the conjunction (AND OCL connector) of all: (i) sub tasks post-conditions; (ii) resource Boolean attributes; (iii) goal Boolean attributes (iv) soft-goal enumerated attributes.
Consider for example, the task To Attract Customers (Figure 2). It is decomposed into three sub-tasks (Delivery, Maintain Stock and Sales By Web) and two subgoals (Good Price and Friendly staff). Let us use the OCL assertions pre-Subtask and pos-Subtask to indicate generic pre and post-conditions of a sub-task. Moreover, assume that the OCL assertion value indicates one of the possible values of the enumerated type (posit, negat, undef,) associated to a soft goal. Figure 4 shows the corresponding OCL description.

\section{Store:: To Attract Customers \\ pre: pre-Delivery and pre-Maintain Stock and pre-Sales By Web \\ post: GoodPrice $=$ 'value' and Friendly staff $=$ 'value' and \\ post-Delivery and post-MaintainStock and post-Sales By Web}

Figure 4: Task decomposition in OCL

When we work with the later requirements we can refine the pre and post conditions of the three operations: Delivery, Maintain Stock and Sales by Web. This activity is typical of later phase of the development process. Hence, we can suggest some conditions for these operations as you can see in the Figure 5. 


\section{Store:: To Delivery \\ pre: delivery address and payment \\ post: delivered CD \\ Store:: To Maintain Stock \\ pre: quantity >= "value" \\ post: $\mathrm{CD}$ in stock \\ Store:: To Sales By Web \\ pre: site on line \\ post: increaseded sales volume}

Figure 5: Some Pre and Post Conditions

The SR models also provides for several types or means-end link. The "end" can be a goal, task, resource, or softgoal, whereas the "means" is usually a task (GT, TT, RT and ST links). Sometimes it is also useful to have means-end hierarchy of softgoals or goals (SS and GG links).

\section{Guideline G6:}

Means end-analysis is represented by OCL disjunctions of all possible means achieving the end.

Guideline G6.1(SS and GG Links):
If the end is a (soft) goal and the means are (soft) goals than the disjunction of the means values implies the end value.

In our case study (see Figure 2), there are two means of offering reasonable prices Negotiating discounts based on high volume purchase (softgoal Volume Purchase[High]) or by promoting sales (softgoal Discount Price). Either way the end goal (softgoal Good Price) is achieved. In Figure 6 the corresponding OCL representation is presented.

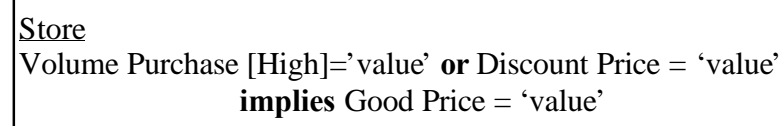

Figure 6: Means-end analysis

\section{Guideline G6.2 (GT, RT and ST Links):}

If the end is either a goal, resource or softgoal and the means is a task than the post-condition of the means task implies the value of end goal (boolean) attribute, resource (boolean) attribute or softgoal (enumerated) attribute.

In our case study these means-end links did not occur.

\section{Guideline G6.3 (TT Link):}

If the end is a task and the means are tasks then the disjunction of the post-condition of the means task imply the pos-conditions of the end task.

In our case study this means-end link did not occur.

Of course not all concepts captured in the early requirements phase will correspond to software system models. The models do not have a one-one relationship; many elements of the organisational model are not part of the software model, since not all of the organisational tasks require a software system. Many tasks contain activities that are performed manually outside the software system, and so do not become part of the software system model. Likewise, many elements in the software model comprise detailed technical software solutions and constructs that are not part of the organisational model. Nonetheless, as we shall see, pUML/OCL also can be used to represent this information.

So far we have been able to identify a context class diagram for problem at hand. Now we can proceed to give some more details of the software system to be developed.

\section{Some Late Requirements}

Late requirements analysis results in a requirements specification document which describes all functional 
and non-functional requirements of the system-to-be. In our case study, the SmartCD actor represents the information system that will help the store to accomplish some of its tasks strategic objective, namely to attract customers (see Figure 2). The system can be structured into various modules or sub-actors including: inventory, financial and the internet sales module.

Due to space limitation we will concentrate on some late requirements for the Internet Sales Module, since it is related to the Sales By Web task, shown in the strategic rationale model of Figure 2. It is part of the system responsible for the process of sales through the Internet. We will consider the context class diagram of Figure 3 as the starting point of our discussion, which by no means should be considered exhaustive. On the contrary, it is meant to be suggestive of the much more complex sets of issues typically found in actual situations. As a matter of fact, methods such as Catalysis [13] already make some of these issues very clear.

The Internet Sales sub-system provides a web-site to enable customers to remotely access the Store. This site allows the visitors to search for CDs, see information about pop stars and news about the musical events.

There are two ways for a client to search for a CD: the fast and super search. In the fast mode the visitor informs the name of the album, of the artist, or of the music. In the super search the objective is to help those that still do not know which title to buy. For that super search type the following options are available: name of the album or of the music, the styles (Pop, Rock, Rap, Reggae, Jazz, etc.), the recording, the repertoire (national or international) and the release time.

Assistance can be provided upon request by e-mail or through a FAQ page. The FAQs (Frequentely Asked and Questions) contains answers for the most common questions. If the visitor does not find the appropriate answer he/she can fill in a form (including the subject, name, number of the purchase request) and submit it. Upon receipt, an on-line sales assistant will answer the question.

A visitor (if not already a client) would have to register in order to use the system. The register operation is available through a page where the customer supplies its personal data (complete name, identification no., gender, birthday), an access name (login), a password, a note (to help to recollect the password), its complete address (street, number, neighborhood, city, state and zip code), residential and commercial telephone, e-mail, the address for delivery (street, number, neighborhood, city, state, zip code, telephone for contact) and the payment form. In particular we are conceiving two forms of payment: credit card or direct debit in checking account. For the payment by credit card it is necessary the titleholder's name, the type of the credit card, the number of the card and validity of the card. For the direct debit in checking account it is necessary the title-holder's name, the name of the bank, the number of the agency and the number of the checking account. If necessary the client can later revise his/her personal information. For reasons of security the customer can choose to fax or e-mail the personal information.

The site offers to its clients special services, for example: security in the transactions and personalized attendance. For the sake of total security the customers' data are stored in a safe and isolated server (not connected to the Internet), with restricted access to authorized employees. SmartCD will use a safe communication protocol (SSL - Secure Sockets Layer). Client profile can also be provided. It consists of client's preferences such as musical styles (Pop/Rock, Blues/Jazz, Infantile, Samba/Pop,...), artists or favorite groups and the desire or not of receiving information on promotions, releases, and so on.

Based on these late requirements a revised pUML class diagram can be drawn (see Figure 7). It is interesting to note that the security[Acess] softgoal between the Client and SmartCD actors of Figure 2 is the reason for the choice of the use of a secure protocol (SSL) for credit and debit operation of Payment Form Class.

\section{Related Work}

The area of Requirements Engineering [14] has developed several novel techniques for early requirements capture $[15,16]$. Bubenko emphasizes the need to model organizations and their actors, motivations and reasons [16]. In his work, enterprise modeling and requirements specification are based on the notion that a requirements specification process, from a documentation point of view, implies populating (instantiating) five interrelated sub-model, representing areas of knowledge of the organization, which include an Objectives Model, an Activities \& Usage Model, an Actors Model, a Concepts Model, and an Information Systems Requirements Model. Since the models are informal, or at best semiformal, only some verification can be performed automatically, such as syntactical correctness and connectedness. 


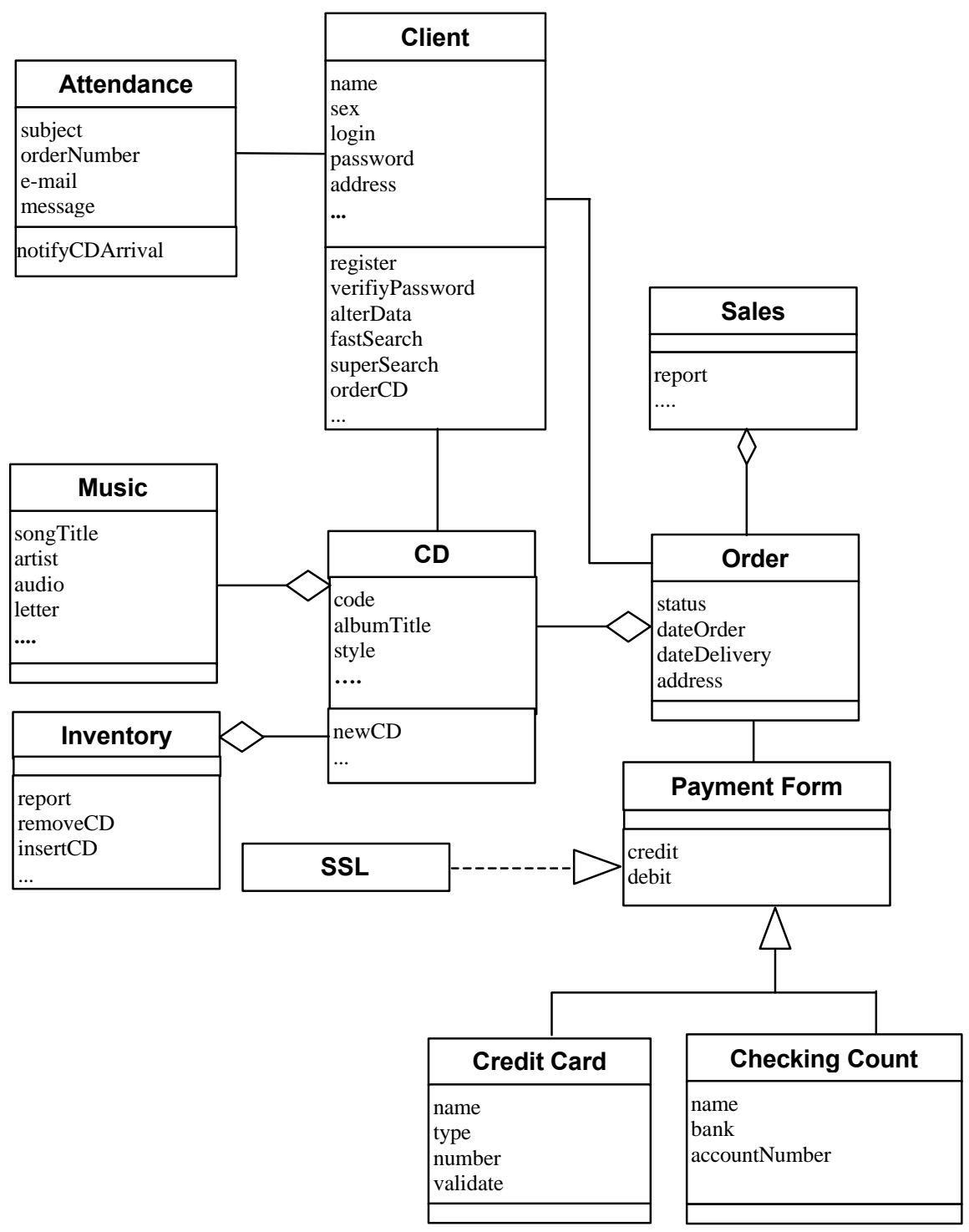

Figure 7: Revised Class Diagram (Internet Sales Sub-System)

In the KAOS framework [16] goals are explicitly modeled and simplified (reduced) through means-end reasoning until it reaches the agent level of responsibilities. KAOS provides a multi-paradigm specification language and a goal-directed elaboration method. The language combines semantic nets for conceptual modeling of goals, requirements, assumptions, agents, objects and operations in the system; temporal logic for the specification of goals, requirements, assumptions and objects; and state-based specifications for the specification of operations. Goals are reduced through meansends reasoning to arrive at responsibilities for agents. The modeling of agents is specificational and prescriptive. Since agents are assumed to conform to prescribed behavior, one cannot easily analyze dependencies for opportunities and vulnerabilities. On the other hand, i* models offer a number of levels of analysis, in terms of ability, workability, viability and believability. These are detailed in [3].

The TROPOS project $[17,18]$ is developing a methodology for agent-oriented systems which is requirements-driven. It adopts the $\mathrm{i}^{*}$ concepts and uses it all along the life-cycle as a foundation to model late requirements, architectural, detailed design and implementation. That allows dealing with intention-based software units at the right phase and not freeze them earlier in the process.

Another related work is the requirements modeling framework for manufacturing systems (MS) presented in [19]. It relies on two major ideas: a multi-formalism approach, combining several languages into a coherent 
formalism, and a component-based modeling approach. The modeling framework proposed combines the Albert II, $i^{*}$ and CIMOSA languages. The combination is achieved through meta-modeling and the definition of a set of mapping rules that establish a correspondence among some of the concepts of the three formalisms.

Another important issue related to early phase requirements capture is the representation of qualities attributes, such as accuracy, performance, security, modifiability, etc. In [12] a comprehensive approach for dealing with non-functional requirements - NFR is presented. Structured graphical facilities are offered for stating NFRs and managing them by refining and interrelating NFRs, justifying decisions, and determining their impact. A current research topic is the extension of traditional Object-Oriented Analysis to explore the alternatives offered by the non-functional goal-oriented analysis, which systematizes the search for a solution which characterizes early phases or requirements analysis, rationalizes the choice of a particular solution, a relates design decisions to their origins in organisational and technical objectives.

Although UML has been used mainly for modeling software, recent proposals have used it for describing enterprise and business modeling. For example, [1] claims that UML is a suitable language for describing both the structural aspects of business (such as the organization, goal hierarchies, or the structure of the resources), the behavioral aspect of a business (such as the processes), and the business rules that affect structure and behaviour. In [20] UML is used, from a business perspective, to describe the four key elements of an enterprise model: purpose, processes, entities and organization. The challenge is to transfer the information available in the (early) business models to the (late) software requirements models.

\section{Conclusion}

In this paper, we have suggested that requirements capture has to be done at different levels of abstraction (ranging from the early phase to the late phase requirements). Furthermore, we argue that UML alone is not adequate to deal with all different types of analysis and reasoning that are required during the requirements capture phases. Instead, we advocate the use of two complementary modelling techniques, $\mathrm{i}^{*}$ and a precise subset of UML.

To model and understand issues of the application and business domain (the enterprise) a developer can use the $i^{*}$ framework which allows a better description of the organisational relationships among the various agents of a system as well as an understanding of the rationale of the decisions taken. For later requirements capture we suggest the use of pUML, a subset of UML which has a well defined semantics. Annotations in OCL can also be deployed for describing constraints on the models.

We believe that each language has its own merits for supporting requirements capture. But as long as different techniques are used, then a key issue is the development of an integrated framework to support and guide the interplay of requirement captures activities at the various levels, and to support traceability and change management. Indeed, the guidelines presented in the paper are important steps in this direction. They can help to map the descriptive, early requirements model of the $i^{*}$ technique into a prescriptive, late requirements model expressed in pUML/OCL.

Of course not all concepts captured in the early requirements phase will correspond to software system models. The models do not have a one-one relationship; many elements of the organisational model are not part of the software model, since not all of the organisational tasks require a software system. Many tasks contain activities that are performed manually outside the software system, and so do not become part of the software system model. Likewise, many elements in the software model comprise detailed technical software solutions and constructs that are not part of the organisational model. Nonetheless, pUML/OCL also can be used to represent this information.

Further research is still required to handle some structuring concepts found in the $i^{*}$ framework, such as agent, role and position. Some real industrial case studies are also expected. Work is underway to provide some tool support (Multiview+) for the mapping. The tool will import organizational requirements specification produced by the $\mathrm{i}^{*}$ toolset (OME) and generate the corresponding pUML business model.

\section{Acknowledgements}

The authors are grateful to John Mylopoulos whose insights helped us focus our research on intentional and social aspects. He provided valuable comments on earlier drafts of this paper. We would like also to thank the anonymous referees for their helpful comments to this work. 


\section{References}

[1] H. Erikson, M. Penker. Business Modeling with UML: Business Patterns at Work, John Wileys \& Sons, Inc., USA, 2000.

[2] I. Jacobson, G. Booch, J. Rumbaugh. Unified Software Development Process, Rational Software Corporation. Addison-Wesley Object Technology Series, 1999.

[3] E. Yu. Towards Modelling and Reasoning Support for Early-Phase Requirements Engineering. In Proc of IEEE International Symposium on Requirements Engineering - RE97, pages 226235, 1997.

[4] G. Booch, I. Jacobson, J. Rumbaugh. Unified Modeling Language User Guide. Rational Software Corporation. Addison-Wesley Object Technology Series, 1999.

[5] J. Mylopoulos, L. Chung, E. Yu. From ObjectOriented to Goal-Oriented Requirements Analysis. Communications of the ACM, 42(1): 31-37, 1999.

[6] E. Yu, J. Mylopoulos. Understanding 'Why' in Software Process Modeling, Analysis and Design. In Proc. Sixteenth International Conference on Software Engineering, Sorrento, Italy, 1994.

[7] A. Evans, S. Kent. Core Meta-Modelling Semantics of UML: The pUML Approach. UML'99 - The Unified Modeling Language. Proc. of <<UML>>'99 The Unified Modeling Language: Beyond the Standard - The Second International Conference. Eds. Robert France and Bernhard Rumpe. Fort Collins, CO, USA, pages 140-15, 1999.

[8] J. B. Warmer, A. G. Kleppe. The Object Constraint Language: Precise Modeling with UML. Addison-Wesley Object Technology Series, 1999.

[9] E. Yu. Why Agent-Oriented Requirements Engineering. In Proc. of the 4th International Workshop on Requirements Engineering: Foundations of Software Quality, Pisa, Italy. E. Dubois, A.L. Opdahl, K. Pohl, eds. Presses Universitaires de Namur, pages 15-22, 1998

[10] F. M. R. Alencar. Mapping Organizational Modeling into Precise Specification. Ph.D. Thesis (In Portuguese). Centro de Informática,
Universidade Federal de Pernambuco, Recife, Brazil, Dec., 1999.

[11] Precise UML Group, pUML: http/www.cs.york.ac.uk/puml, Out. 2000.

[12] L. K. Chung, B. A. Nixon, E. Yu, J. Mylopoulos. Non-Functional Requirements in Software Engineering, Kluwer Publishing, 2000.

[13] D. F. D’Souza, A. C. Wills. Objects, Components, and Frameworks with UML: The CATALYSIS Approach. Addison-Wesley Object Technology Series, 1999.

[14] A. van Lamsweerde. Requirements Engineering in the year 00: A Research Perspective. Invited paper to ICSE'2000. In Proc. $22^{\text {nd }}$ International Conference on Software Engineering, Limerick, 2000.

[15] M. Boman, J. Bubenko, P. Johannesson, B. Wangler. Conceptual Modeling. Prentice Hall Series in Computer Science, 1997.

[16] A. van Lamsweerde, R. Darimont, E. Letier. Managing Conflicts in Goal-Drivel Requirements Engineering. IEEE Transaction on Software Engineering, Special Issue on Inconsistency Management in Software Development, 1998.

[17] J. Castro, M. Kolp, J. Mylopoulos. Developing Agent-Oriented Information Systems for the Enterprise. In Proc of the Second International Conference On Enterprise Information Systems (ICEIS00), Stafford, UK, 2000.

[18] J. Mylopoulos, J. F. B. Castro. Tropos: A Framework for Requirements-Driven Software Development. Brinkkemper, J. and Solvberg, A. (eds.), Information Systems Engineering: State of the Art and Research Themes, SpringerVerlag, pages 261-273, 2000.

[19] M. Petit. Formal Requirements Engineering of Manufacturing Systems: A Multi-Formalism and Component-Based Approach. Ph.D. Thesis, Computer Science Departament University of Namur, Namur, Belgium, 1999.

[20] C. Marshal. Enterprise Modeling with UML: Designing Successful Software through Business Analysis. Addison-Wesley Object Technology Series, 2000. 